\title{
How students apply linear algebra to quantum mechanics
}

\author{
Charlotte Hillebrand-Viljoen ${ }^{1}$ and Spencer Wheaton ${ }^{1}$ \\ ${ }^{1}$ Department of Physics, University of Cape Town, University Avenue, Cape Town, Western Cape, South Africa
}

This paper describes a framework for how students apply linear algebra to quantum mechanics based on a survey of 61 students at various levels. The framework divides mathematics into calculational and valueassigning parts in contrast to storytelling parts, which are concerned with the relationships between objects. We apply this to student understanding in the context of linear algebra and quantum mechanics, and give some implications for instruction.

\section{INTRODUCTION}

Most people use simple mathematics on a regular basis, but physicists, however, seem to have an especially intimate relationship with the subject. This gives rise to such aphorisms as "mathematics is the language of physics." The use of mathematics as a language in physics is discussed in, e.g. [8]. This kind of mathematical activity is an important component of a physicist's toolbox and something that physics students need to learn. Previous studies have focused on students' use of high school level algebra (e.g. [5], [6]) and advanced calculus (e.g. [9]) in physics.

Another area of mathematics that is important for physics is linear algebra. Linear algebra is usually students' first encounter with abstract, proof-based mathematics [1]. Quantum mechanics in particular makes use of the principles of linear algebra in a more fundamental and explicit way than most other intermediate physics courses. (For example, Griffiths' textbook [2] devotes an entire chapter to the mathematical formalism of quantum mechanics.) Previous reviews of student difficulties with quantum mechanics (e.g. [10]) have presented several common difficulties which could be related to the formulation of qunatum mechanics linear algebra. We have thus chosen to study students' applications of linear algebra to quantum mechanics.

We have developed a general framework which describes the application of linear algebra to quantum mechanics in terms of "math stories" and "physics stories': concepts which can be applied more widely. After describing the methodology in Section II, we describe the framework in Section III below. In Section IV, we discuss several applications of the framework to student understanding, looking at stories students tell about vectors, students' difficulties engaging with math stories and student views of how linear algebra applies to quantum mechanics. We discuss implications for instruction along the way. Section $\mathrm{V}$ summarizes the work so far and describes work still to be done.

\section{RESEARCH METHODOLOGY}

Our study takes a Grounded Theory approach, following the work of Corbin and Strauss [3]. This is a qualitative methodology which emphasises the grounding of theory and high-level description in empirical data.
To obtain our data, we surveyed sixty-one university physics students of different levels. These students ranged from second year university students who had completed a course in linear algebra, but not begun quantum mechanics, to gradiate students conducting research in related areas. Students were asked to give free-written responses to a number of questions about quantum mechanics (or physics in general where appropriate), linear algebra and their attitudes to these subjects.

These data were analysed through an extensive process of memo writing, following the methodology of Corbin and Strauss. Memos were used to develop indices and codes, with descriptions and typifying quotes agreed on between the researchers. This scheme was used to develop the framework below, which aims to orient discussion of student applications of linear algebra to quantum mechanics, for use as in Section IV.

\section{FRAMEWORK}

The relationship between physics and mathematics has been described in the literature [4] as a mapping from stories about physics to symbolic mathematical equations. Here, we refine this type of description and consider the mathematical and physical aspects in more detail. We introduce the idea of a mathematical story as distinct from a calculation, which we believe synergizes with the theory of symbolic forms [5].

We consider math-stories to be that part of mathematics which is concerned with defining objects and their relationships; and following through to observe the natural consequences of these relationships. (Replacing "defining objects" with "describing characters" gives a more literary description of a story and may serve to explain the choice of terminology.) The prototypical math-story is the formal proof, which begins with a set of definitions and relationships and ends by showing that some interesting result is the logical consequence of the given set up. However, math-story thinking can be less formal and so we more generally refer to the activity of telling math-stories.

On the other hand, calculational activities are those which are concerned with carrying out a set of steps or procedures to achieve a desired result. Calculations are generally informed by math stories (although the calculator may not be aware of this), but the focus is on finding a value, rather than exploring 
and describing relationships. In this sense the calculation is very much distinct from the math story.

Student descriptions of linear algebra, and particularly discussions of what makes it difficult or what makes it applicable, tend to be either calculation-focused or story-focused; or in some cases, to demonstrate an awareness of both kinds of thinking. An example of this from the survey is a student who writes, "Though I am not particularly adept at matrix algebra, my intuition for proofs of statements in linear algebra is generally good." Here, the student suggests that their skills at telling math stories are greater than their calculational skills. Another student has an opposing opinion about math stories, writing, "The aspects [of linear algebra] I did not enjoy were learning the definitions [and] theorems." An example of a student considering only the calculational aspect of linear algebra is the one who writes, "I don't think I learnt any important skills in linear algebra. I was quite comfortable working with matrices from what I had learned in applied math and physics." A counterpoint, from the same student cohort, is "I've seen it [linear algebra] appear in many different aspects of my courses in Applied Maths and Physics and they don't go in deep to explain the basics." These examples also exhibit the strong feelings that students often have about different aspects of linear algebra. We will discuss students' difficulties with telling math stories in Section IV B.

In parallel with the division of mathematical thinking into telling math stories and calculational thinking, we divide physics thinking into the telling of physics stories (which involve physics entities, their relationships and the natural consequences of those) and the assigning of values to physical quantities. Note that assigning values to physical quantities may involve measuring, estimating or calculating, but it is not directly concerned with considering the interactions between physical objects. In general this assigment is informed by an underlying physics story, but as in the case of mathematical calculations, the assignment could be carried out without an underlying understanding (the infamous "plug and chug" approach). A student view of this distinction is encapsulated in the comment that, "Physics involves a lot of understanding the theory behind things as opposed to just knowing how to apply it." This framework is summarized in Fig. 1.

\section{APPLICATIONS}

\section{A. Stories about the wavefunction vector}

A primary way of investigating student understanding within this framework is to look at the stories that students tell. Here we consider the stories students tell about vectors that represent wavefunctions. We observe three classes of descriptions for wavefunction vectors in the data: the vector as a quantity with magnitude and direction, the vector as an element of a vector space, and the vector as a linear combination of basis elements. The last of these is predominantly used for telling math stories, which suggests that explicitly teaching

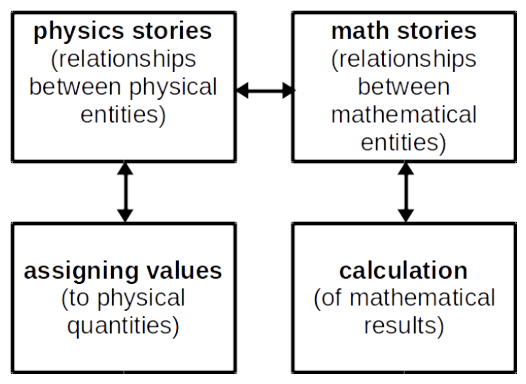

FIG. 1. In understanding how students relate mathematics and physics, we find it useful to divide mathematics into a calculational part (bottom right), which is primarily concerned with carrying out procedures to obtain values and a story part, which is primarily concerned with the relationships between mathematical entities. Similarly, physics is divided into assigning values to physical quantities and telling the stories which express the relationships between physical entities. Math and physics are connected via their respective stories, although solving a problem may also involve performing calculations and assigning values.

students to use this kind of description may aid their conceptual understanding.

The first category of description is usually expressed either by drawing the vector as an arrow or by stating that, "A vector is a quantity with magnitude and direction." This is a common description of a vector in high school and introductory physics courses, and it is much more popular amongst relatively junior students in our sample. This view is commonly seen in concert with the assumption that all vectors are elements of Euclidean space. In our data, students rarely used this Euclidean description to explain concepts from quantum mechanics. Those who attempted to do so did not produce consistent descriptions.

The second category of description that is often used for the wavefunction vector is the abstract definition of the vector as an element of a vector space. This is mathematically correct, although it relies on the full definition of a vector space. This definition is often used to tell simple stories. For example, "A vector is an element of a vector space and satisfies certain properties [...] a 'wavefunction is a vector' means the wavefunction also satisfies these properties." Some students also used this definition to tell more complicated stories such as, "By its very definition, a basis will always be able to describe a vector in a certain vector space. Since wavefunctions are vectors in Hilbert space, [if] the eigenstates are a basis for Hilbert space, then the wavefunction will always be able to be expressed in terms of them." Other students simply give up when asked to tell such complex stories, making comments like, "I don't really understand it," while a further set transition to a linear combination view as things get more complex. For example, one student states at this point that "A wavefunction and it's [sic] expansion into a linear combination of eigenstates are the same vector."

When students use the linear combination view explicitly, they often describe the wavefunction vector by writing out 
a mathematical expression for a sum of vectors with scalar coefficients. Sometimes they also describe the wavefunction as a column vector where the vector components are the coefficients of the linear combination. Of course, this is not a fundamental definition, but it is correct for any vector space on which a basis has been defined. Physicists almost always assume the existence of a basis, to the extent that introductory students are widely taught to write Euclidean vectors in terms of the basis elements $\hat{\mathbf{x}}, \hat{\mathbf{y}}$ and $\hat{\mathbf{z}}$.

We observe that students who use the idea of the vector as a linear combination to tell stories about expansion in stationary states do not always consider this description when asked to describe a vector in as many ways as possible (although others do). It seems likely that something about the topic prompts them to consider this new description. We surmise that many students who cannot tell a consistent story about expansion in stationary states do not consider the linear combination description of a vector at all. Because this description seems to be helpful to many students in constructing math stories, we conjecture that explicit instruction in this way of viewing the wavefunction vector will improve students' conceptual understanding of quantum mechanics. Additionally, highlighting the connection between the basis vectors and the $\hat{\mathbf{x}}$ notation may provide some familiarity to students who are overwhelmed by the large amount of new notation introduced in quantum mechanics.

\section{B. Student difficulties with telling math stories}

Following the structure of the framework described in Fig. 1 , one can imagine that a typical problem-solving exercise might involve identifying a quantity of interest, to which we wish to assign a value (bottom left), telling a physics story about this quantity (top left) which suggests a corresponding math story (top right) and so performing a calculation suggested by this math story (bottom right) which produces the value we are looking for. Of course, students do not always follow this pathway. A popular alternative is the "plug and chug" approach to problem solving, in which students find an equation that seems to match the problem statement, do some calculations, and present a result, bypassing the story part of the diagram altogether. It is possible to design a question that clearly distinguishes approaches using these routes, such as a question in which the calculational part is trivial if the math story is understood, but requires some working otherwise. Although they do not use this terminology, Kuo et al. [6] have carried out such a study, using the language of symbolic forms, and shown that students can be encouraged to consider the math and physics stories involved in a problem with appropriate prompting. . In our data we uncover a somewhat more subtle situation, in which students take the physics story into consideration, but struggle to deal with the math story. One student says "I find it difficult to remember to connect the macro use of it [linear algebra] in quantum to the theory and conceptual understanding of it." Another student makes the somewhat conflicted statement that, "Understanding the ideas and concepts behind what you're doing is important, but some of it isn't that important," going on to say, "I don't think that it is important to have to memorize all the proofs." This view of proofs as an exercise in memorization is fairly common, but is not well aligned with the idea of proofs as math stories.

Storytelling may require some memorization, to ensure that all the story's key events are covered, but it is not fundamentally an exercise in memorization. Rather, it is concerned with understanding the relationships between objects and the natural development of those relationships. If students are resorting to memorizing proofs verbatim, it is likely that they are not comfortable working with the relationships between mathematical objects. In other words, they are not comfortable telling math stories.

In introductory physics courses, students only need to be comfortable with relatively simple, intuitive math stories. An intuition for arithmetic operations is well ingrained at school level (certainly for most students studying physics at a tertiary level) and the basics of integro-differential calculus can largely be understood in terms of these, with the addition of limits. Linear algebra introduces an entirely new set of ideas, such as linear independence and span, as well as greater reliance on formal proofs which students often struggle to comprehend [1,7]. Ideally, students would learn to tell stories about these concepts in the first linear algebra course, as is the case for a student who comments that, "[The big ideas of linear algebra] are important, even just for the sake of that kind of thinking." However many students are much less comfortable, to the extent of one student who comments, "I don't think it [referring to "things like vector spaces etc.'] is important, I have not found a use for it," at the end of the quantum mechanics course.

This lack of mathematical understanding must have an impact on conceptual understanding of physics topics like quantum mechanics. We see this, for example, in students' explanations of the expansion of a wavefunction in terms of stationary states. These rely heavily on math stories about eigenstates, when students give them at all. A number of students who express discomfort with telling math stories do not even attempt to explain this idea.

We would argue that it is crucial for instructors to be aware of students' discomfort with math stories. Even students who are aware of the importance of math stories in quantifying physical relationships may find it difficult to make the connection between the math and the physics, as with the student quoted earlier who says "I find it difficult to remember to connect the macro use of it in quantum to the theory and conceptual understanding of it." This suggests that it would be beneficial for instructors to explicitly and repeatedly draw the connections between the physics stories and math stories they are telling in class, and to explicitly emphasize the importance of some level of engagement with the math stories related to the content. 


\section{Student views of the relationship between linear algebra and quantum mechanics}

We can also explore student attitudes to math stories in physics by looking at why they say linear algebra is applicable to quantum mechanics. (In principle not all students would be expected to say linear algebra is applicable at all, but all students in our sample took this stance.) Attitudes can be grouped into three major categories.

The first of these is well represented by a student who wrote "I have been convinced it is [useful]." Students in this category know the accepted view is that linear algebra is important, but have not reached that conclusion for themselves. Unsurprisingly, this attitude was more common in students who had not yet studied quantum mechanics, although it was not unique to them.

A second category comprises those who have made an implicit connection between the ideas of quantum mechanics and linear algebra, but do not explicitly articulate the idea of a mapping between the subjects. A typical student in this category says that, "only through quantum mechanics [...] did I see how it [linear algebra] might be applied." Also typical of this category are students who give lists of examples where linear algebra is applied to quantum mechanics. These students do not talk about the intimate connection between the stories of linear algebra and the stories of quantum mechanics. Although an understanding of these stories may underlie particular examples, the universal application of these stories is not recognized.

The third category, largely featuring graduate students, consists of students who explicitly recognize a mapping between the stories of quantum mechanics and linear algebra. This view was succinctly expressed by a student who wrote, "any problem that can be mapped onto a linear algebra problem is essentially solved." Students in this category typically talk about ideas like mappings and structure in explaining why linear algebra is broadly applicable to quantum mechanics (and elsewhere).

An interesting meta-feature of this analysis was the criticism that many students expressed with the inadequacy of their responses. While students seemed comfortable leaving out questions on more technical topics, or even giving flippant responses such as, "Isn't that your job?", several students were unhappy about their responses in this section. One stu- dent, after crossing out several lines of writing, said "Thankfully I am not the only person answering such a questionnaire. Hopefully others will fill in all the gaps I miss." Another commented "It's difficult for me to formulate a more precise answer than that, I'm afraid." This is perhaps indicative of the fact that students are rarely required to deal with metacognitive tasks on any explicit level. We saw in Section IV B that being comfortable with math stories is important to success with conceptual physics, and that many students consider math stories to be unimportant. This discomfort with metacognition may be an additional indicator that explicitly talking about the utility of the stories of linear algebra in quantum mechanics is beneficial to students.

\section{v. CONCLUSION}

We have laid out a framework describing ways students relate linear algebra to quantum mechanics, and more generally how students relate mathematics to physics. This framework focuses on telling stories in math and physics spaces (in addition to performing calculations and assigning values) as important skills. We also see that students are often not comfortable with storytelling skills; and especially (at this level) with telling math stories. We suggest that explicit instruction in the importance of these stories and in relating them to physics may improve students' conceptual understanding of quantum mechanics. We also discussed different ways that students tell stories about the wavefunction vector and suggested that explicit instruction in some of these may be beneficial to students' conceptual understanding.

Future work on this topic will involve applying the coding scheme to the data in more detail, to acquire quantitative results regarding the number of students in various categories and their overlaps. We will also look at applying existing theories from cognitive science to better describe the relationship between math and physics stories.

\section{ACKNOWLEDGMENTS}

$\mathrm{CH}$ acknowledges financial support from the NRF of South Africa and the UCT Science Faculty.

\section{(2013)}

[7] D. Plaxco and M. Wawro, The Journal of Mathematical Behaviour 38 (2015)

[8] E.F. Redish and E. Kuo, Sci \& Educ 24 (2015)

[9] M.B. Kustuch, D. Roundy, T. Dray and C.A. Manogue, Phys. Rev. ST Phys. Ed. Res. 10, 1 (2014)

[10] C. Singh and E. Marshman, Phys. Rev. ST Phys. Ed. Res. 11, 2 (2015)

2 (2007)
[5] B.L. Sherin, Cogn. Instr. 19, 4 (2001)

[6] E. Kuo, M.M. Hull, A. Gupta and A. Elby, Sci. Educ. 91, 1 